\title{
Pharmacokinetics and Safety of Duloxetine Enteric-coated Tablets in Chinese Healthy Volunteers: A Randomized, Open-label, Single- and Multiple-dose Study
}

\author{
Huafang $\mathrm{Li}^{*}$, Ting $\mathrm{Li}^{*}$, Yan Li, Yifeng Shen \\ Shanghai Mental Health Center, Shanghai Jiao Tong University School of Medicine, Shanghai, China
}

\begin{abstract}
Objective: Duloxetine hydrochloride is a selective serotonin (5-hydroxytryptamine) and norepinephrine reuptake inhibitor. It is approved for effective treatment for major depressive disorder. The pharmacokinetics (PK) of duloxetine has been studied, but few pharmacokinetics properties in Chinese subjects are available. This study explored the dose proportionality and determined duloxetine levels in human plasma by comparing the PK properties after administration of single or multiple doses in healthy volunteers.

Methods: Thirty-six subjects were divided randomly into three groups and received a single dose of 15, 30, or 60 mg duloxetine. Those who received $30 \mathrm{mg}$ continued on to the multiple-dose phase and received $30 \mathrm{mg}$ daily for 7 days. Liquid chromatography/mass spectroscopy was applied to determine concentrations. The PK properties were calculated and included maximum plasma concentration $\left(\mathrm{C}_{\max }\right)$, time when maximum plasma concentration was reached $\left(T_{\max }\right)$, time when half-maximum plasma concentration was reached $\left(t_{1 / 2}\right)$, area under the plasma concentration-time curve from time zero to the last measurable concentration $\left(\mathrm{AUC}_{0-\uparrow}\right)$, mean concentration levels $\left(\mathrm{AUC}_{0-\infty}\right)$, and apparent total clearance of the drug from plasma after oral administration (CL/F).

Results: The standard calibration curve was linear in the concentration range 0.11-112 ng/ml ( $r>0.992)$. Linear PK properties were found at doses of $15-60 \mathrm{mg}$. The $C_{\max }$ and AUC were proportional to dose, but the $T_{\max }$ and $t_{1 / 2}$ did not increase with increasing dose.

Conclusion: No significant differences in the PK parameters were found among the three groups during the single-dose phase. The $\mathrm{AUC}$ and $\mathrm{C}_{\max }$ were greater in the multiple-dose phase, indicating duloxetine accumulation following multiple-dose administration.
\end{abstract}

KEY WORDS: Duloxetine; Pharmacokinetics.

\section{INTRODUCTION}

Duloxetine hydrochloride, (+)-(s)-N-methyl-(naphtyloxy)-2-thiophenepropylamine hydrochloride, is a selective serotonin (5-hydroxytryptamine [5-HT]) and norepinephrine (NE) reuptake inhibitor developed by Eli Lily Company. ${ }^{1)}$ Its molecular formula is $\mathrm{C}_{18} \mathrm{H}_{19} \mathrm{NOS} \cdot \mathrm{HCI}$, and the molecular weight is 333.88. Duloxetine enhances 5-HT and NE function in the central nervous system and is

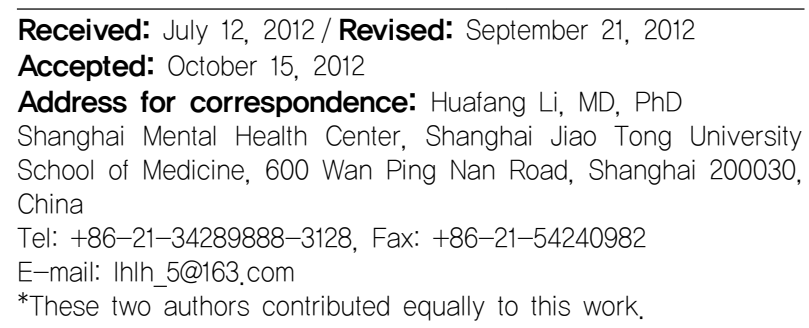

an effective treatment for major depressive disorder (MDD), pain, and stress urinary incontinence. ${ }^{2,3)}$ Duloxetine has low in vitro affinity to dopaminergic, serotonergic, cholinergic, adrenergic, histaminergic, and $\gamma$-aminobutyrate receptors but does not inhibit monoamine oxidase.

Duloxetine is extensively metabolized hepatically by cytochrome P450 (CYP) isozymes 1A2 and 2D6. ${ }^{4,5)}$ It has high affinity for plasma protein and is primarily conjugated with albumin and $\alpha 1$ acid glycoprotein. ${ }^{6)}$ The main biological conversion pathway is combined naphthyl epoxidation and oxidation. The time when half-maximum plasma concentration was reached $\left(\mathrm{t}_{1 / 2}\right)$ is about 12 hours (range, 8-17 hours) and the drug reaches a steady plasma drug concentration within 3 days after initial administration. Maximum plasma concentration $\left(\mathrm{C}_{\max }\right)$ is reached in 6 hours after oral administration, with no effect

(c) This is an Open-Access article distributed under the terms of the Creative Commons Attribution Non-Commercial License (http://creativecommons.org/licenses/by-nc/3.0) which permits unrestricted non-commercial use, distribution, and reproduction in any medium, provided the original work is properly cited. 
of food, but food can delay the peak time to 6-10 hours and decrease the degree of absorption by $10 \%$. If duloxetine is administered at night, absorption lags about 3 hours compared with morning administration, and apparent clearance increases by one-third. The estimated apparent volume of distribution is 1,640 L. $^{7,8)}$ Various clinical trials have indicated that duloxetine is effective for treating MDD at doses of 40-120 mg daily. Common adverse effects are nausea, vomiting, dry mouth, drowsiness, decreased appetite, and increased sweating. No definite clinical data are available to indicate its effect on blood pressure, heart rate, or QT interval.

This study was designed to evaluate the pharmacokinetic (PK) properties and safety of duloxetine in Chinese healthy volunteers after single and multiple-dose administration.

\section{METHODS}

\section{Experimental Methods}

This single-center, open-label study was designed to determine the PK properties of duloxetine after single and multiple-dose administration. Thirty-six subjects were selected to participate from among 44 Chinese volunteers based on medical history, a physical examination, electrocardiogram (ECG), and clinical laboratory screening criteria assessments. The examinations ensured that the participants had no history of cardiovascular, gastrointestinal, respiratory, renal, endocrinal, psychiatric, or nervous system diseases. Subjects were excluded if they had substance or alcohol abuse problems or used any kind of drugs (including Chinese herbal drugs) during 1 week prior to the study.

This study protocol was reviewed and approved by the Institutional Review Board of Shanghai Mental Health Center. Written informed consent was obtained from all subjects prior to any study-related activity.

\section{Study Design}

The study included single and multiple-dose phases. Thirty-six subjects were randomly divided into low, middle, and high dose groups ( $n=12 /$ group) for the single-dose phase, and duloxetine was administered orally once at 15,30 , and $60 \mathrm{mg}$.

All subjects were hospitalized the night before the study and fasted 10 hours before drug administration. All single-dose phase participants received a single dose of 15,30 , or $60 \mathrm{mg}$ duloxetine with $200 \mathrm{ml}$ water at 8:00 AM. Subjects assigned to the duloxetine middle group in the single-dose phase continued to the multiple-dose phase with an interval of 7 days and received $30 \mathrm{mg}$ duloxetine daily for 7 days. Standard meals were provided at 8:30, 12:30, and 18:00, and calorie intake did not exceed 2,200 kcal. No strenuous physical activity was permitted during the 24-hour period after drug administration. Smoking and consumption of alcohol or caffeine-containing beverages were prohibited throughout the study.

In the single-dose phase, sequential blood samples ( 3 $\mathrm{ml}$ each) were collected from an indwelling venous catheter at $0,1,2,4,5,6,8,12,15,24,36,48$, and 72 hours after drug administration. Samples of venous blood for the multiple-dose phase were drawn before drug administration and on days 4,5 , and 6 to determine the minimum steady-state plasma drug concentration during a dosage interval ( $\left.\mathrm{Css}_{\min }\right)$. On day 7 , blood samples were drawn at $0,1,2,3,4,5,6,8,12,15,24,36,48$, and 60 hours after drug administration. All other experimental conditions were the same as those during the single-dose phase.

\section{PK Analysis}

Duloxetine plasma concentrations were detected by liquid chromatography/mass spectroscopy. Individual PK data were analyzed according to the mono-compartmental method. The PK parameters analysis was performed using the SAS 9.1.3 (SAS Institute Cary, NC, USA), DAS 3.0 (NCES, Alexandria, VA, USA), and Winnonlin statistics program (Pharsight, Phoenix, AZ, USA). The following PK parameters were determined for each subject in the single-dose phase: $\mathrm{C}_{\max }$, time to maximum plasma concentration $\left(T_{\max }\right), t_{1 / 2}$, area under the plasma concentration-time curve from time zero to the last measurable concentration $\left(\mathrm{AUC}_{0-\mathrm{t}}\right)$, mean concentration levels $\left(\mathrm{AUC}_{0-\infty}\right)$, apparent total clearance of the drug from plasma after oral administration $(\mathrm{CL} / \mathrm{F})$, and apparent volume of distribution $\left(\mathrm{Vd}_{\beta} / \mathrm{F}\right) . \mathrm{C}_{\max }, \mathrm{T}_{\max }, \mathrm{t}_{1 / 2}, \mathrm{AUC}_{0-\mathrm{t}}, \mathrm{CL} / \mathrm{F}$, and $\mathrm{AUC}_{0-\mathrm{t}}$ on day $7 /$ day 1 were determined for each subject in the multiple-dose phase.

\section{Tolerability Evaluation}

Adverse events were elicited from the subjects by means of spontaneous reporting and specific questioning. Clinical laboratory tests (serum chemistry, hematology, and urinalysis), and physical examinations including vital signs and ECG, findings were recorded.

\section{Statistical Analysis}

Descriptive statistics were used to summarize the duloxetine PK parameters by dose. Untransformed and 
A

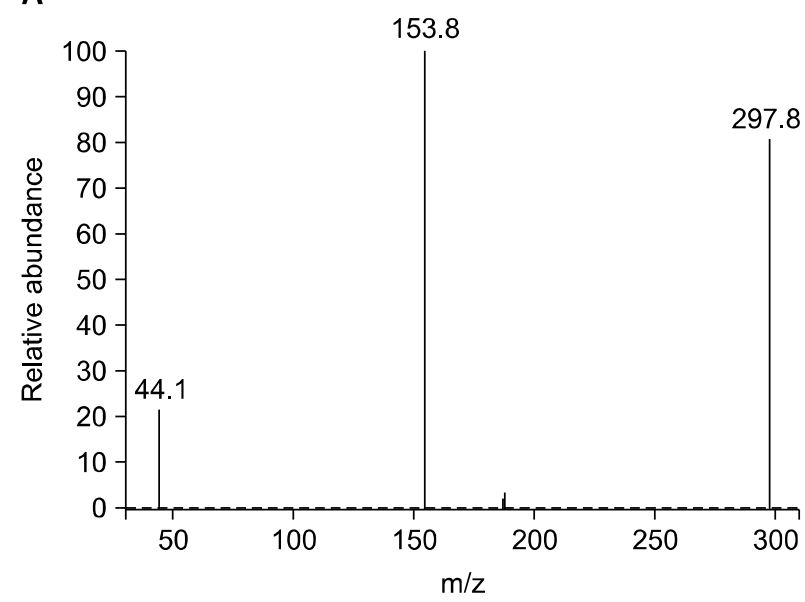

B

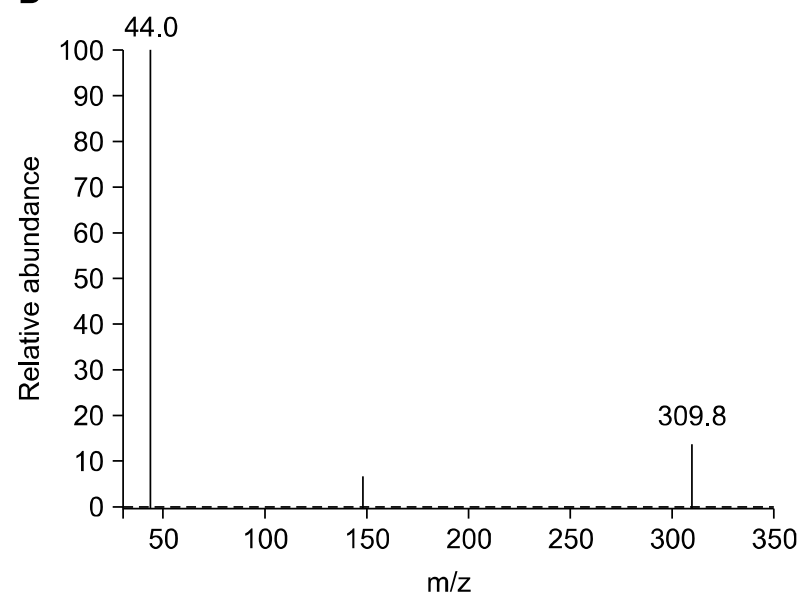

Fig. 1. The selective reaction detection of ion scan mass spectra of duloxetine and the internal standard, fuloxetine. (A) Product ion scan mass spectra of duloxetine. (B) Product ion scan mass spectra of fuloxetine.

log-transformed data for $\mathrm{C}_{\max }$ and $\mathrm{AUC}$ were analyzed using an analysis of variance regression model to establish dose linearity and dose proportionality. The statistical tests were two-sided at the 0.05 level of significance. The $t$-test was used to assess gender differences. The $95 \%$ confidence intervals (CIs) for each difference were calculated.

\section{RESULTS}

\section{Subjects}

Thirty-six healthy volunteers were enrolled in the single-dose phase of the study. Their mean ages were $24.08 \pm 3.15,24.83 \pm 2.62$, and $22.92 \pm 3.26$ years in the low, middle, and high dose groups, respectively. Mean body weights were $58.50 \pm 6.22,57.88 \pm 7.81$, and $59.08 \pm 6.00$ $\mathrm{kg}$, and heights were $165.42 \pm 5.98,165.25 \pm 8.53$, and $163.08 \pm 7.83 \mathrm{~cm}$ in the three groups, respectively.

\section{Method Validation}

The chromatographic results are shown in Fig. 1. The duloxetine retention time was 4.3 minutes. No interference was observed at the duloxetine retention time.

The duloxetine standard calibration curve was linear (range, $0.11-112.00 \mathrm{ng} / \mathrm{ml})(r>0.992)$. The limit of quantification was $0.11 \mathrm{ng} / \mathrm{ml}$ and was defined at a signal-to-noise ratio of $>10$. The methodological and extraction recoveries were $85-115 \%$. The intra-day and inter-day relative standard deviations (RSDs) were $<10 \%$. Stability tests showed that the plasma sample and stock solutions were stable (RSD $<10 \%$; percent different $<10 \%)$ after short-term storage $(0,4,8$, and 12 hours at

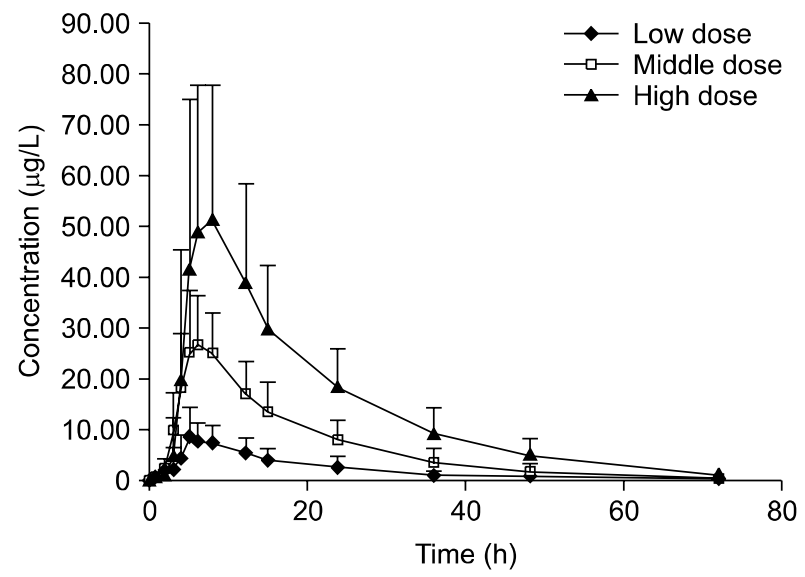

Fig. 2. Mean plasma concentration-time curve after a single oral dose of 15,30 or $60 \mathrm{mg}$ of duloxetine.

$25^{\circ} \mathrm{C}$ and 20 hours at $\left.4^{\circ} \mathrm{C}\right)$, three freeze/thaw cycles $(-20$ $\left.25^{\circ} \mathrm{C}\right)$, and long-term storage $\left(60\right.$ days at $\left.-20^{\circ} \mathrm{C}\right)$.

\section{PKs}

The mean plasma duloxetine concentration-time profiles after a single oral dose of 15,30, or $60 \mathrm{mg}$ are shown in Fig. 2, and the PK parameters are summarized in Table 1. No significant differences in the PK parameters were found among the three dose groups $\left(\mathrm{t}_{1 / 2}: p=0.074 ; \mathrm{V} 1 / \mathrm{F}\right.$ : $p=0.150 ; \mathrm{CL} / \mathrm{F}: p=0.081 ;$ lag time $\left.\left[\mathrm{T}_{\mathrm{lag}}\right]: p=0.502\right)$. In the 15-60 mg range, $\mathrm{C}_{\max }$ and $\mathrm{AUC}$ were proportional to dose $(\mathrm{r}=0.771$ and 0.730 , respectively; $p<0.001)$, and $\mathrm{T}_{\max }$ and $\mathrm{t}_{1 / 2}$ did not increase with increasing dose. Linear PK properties were found for duloxetine enteric-coated tablets at doses of 15-60 mg in vivo (Fig. 3). The slopes of the $\mathrm{C}_{\max }$ and $\mathrm{AUC}_{0-\infty}$ plots were 0.98 and 19.90 , and the $90 \%$ CIs 
Table 1. Pharmacokinetic parameters of duloxetine after a single dose including the 3 dose groups and multiple dosage in Chinese volunteers

\begin{tabular}{|c|c|c|c|c|}
\hline $\begin{array}{l}\text { Pharmacokinetic } \\
\text { parameter }\end{array}$ & $\begin{array}{l}\text { Low dosage group } \\
\text { (15 mg) }\end{array}$ & $\begin{array}{l}\text { Middle dosage group } \\
\text { (30 mg) }\end{array}$ & $\begin{array}{l}\text { High dosage group } \\
\text { (60 mg) }\end{array}$ & $\begin{array}{l}\text { Multiple dosage } \\
\text { (30 mg for } 7 \text { days) }\end{array}$ \\
\hline$T_{1 / 2}(h)$ & $9.52 \pm 3.11$ & $10.19 \pm 2.28$ & $11.89 \pm 2.07$ & $11.39 \pm 2.03$ \\
\hline$T_{\max }(h)$ & $5.67 \pm 1.37$ & $5.75 \pm 1.22$ & $7.33 \pm 1.92$ & $6.50 \pm 1.17$ \\
\hline$C_{\max }(\mu \mathrm{g} / \mathrm{L})$ & $10.44 \pm 4.91$ & $31.82 \pm 7.56$ & $55.88 \pm 29.23$ & $43.09 \pm 16.54$ \\
\hline$K_{e}(L / h)$ & $0.08 \pm 0.03$ & $0.07 \pm 0.02$ & $0.06 \pm 0.01$ & $0.06 \pm 0.01$ \\
\hline $\mathrm{V}_{1 / \mathrm{F}}(\mathrm{L})$ & $1,808.04 \pm 1,455.38$ & $1,029.30 \pm 325.73$ & $1,324.31 \pm 738.71$ & $962.70 \pm 373.98$ \\
\hline $\mathrm{C}_{\mathrm{L} / \mathrm{F}}(\mathrm{L} / \mathrm{h})$ & $149.53 \pm 142.91$ & $73.71 \pm 30.12$ & $79.45 \pm 47.5$ & $59.78 \pm 22.45$ \\
\hline MRT $_{0-\dagger}(h)$ & $16.31 \pm 3.19$ & $16.78 \pm 3.07$ & $20.34 \pm 2.47$ & $17.88 \pm 2.22$ \\
\hline $\mathrm{AUC}_{0-\dagger}(\mu \mathrm{g} / \mathrm{L} \times \mathrm{h})$ & $141.43 \pm 79.06$ & $463.18 \pm 208.24$ & $931.51 \pm 432.24$ & $822.35 \pm 364.31$ \\
\hline $\mathrm{AUC}_{0-\infty}(\mu \mathrm{g} / \mathrm{L} \times \mathrm{h})$ & $175.39 \pm 123.42$ & $489.35 \pm 213.74$ & $1,073.99 \pm 499.37$ & $840.26 \pm 379.54$ \\
\hline$A_{U} C_{s s}(\mu \mathrm{g} / \mathrm{L} \times \mathrm{h})$ & --- & ---- & --- & $584.94 \pm 234.34$ \\
\hline
\end{tabular}

Values are presented as mean \pm standard deviation.

$T_{1 / 2}$, elimination half-life; $T_{\max }$, time to $C_{\max } ; C_{\max }$, maximum observed concentration; $K_{e}$, the rate of elimination; $V_{1 / F}$, initial volume of distribution; $\mathrm{C}_{\mathrm{L} / \mathrm{F}}$, oral system clearance; $\mathrm{MRT}_{0-t,}$, the mean residence time of 0-120 min; AUC 0 -t, area under concentration time curve (AUC) from time zero to definite time; $A \cup C_{0-\infty}, A \cup C$ from time zero to infinity; $A \cup C_{s s,} A \cup C$ in steady-state.

A

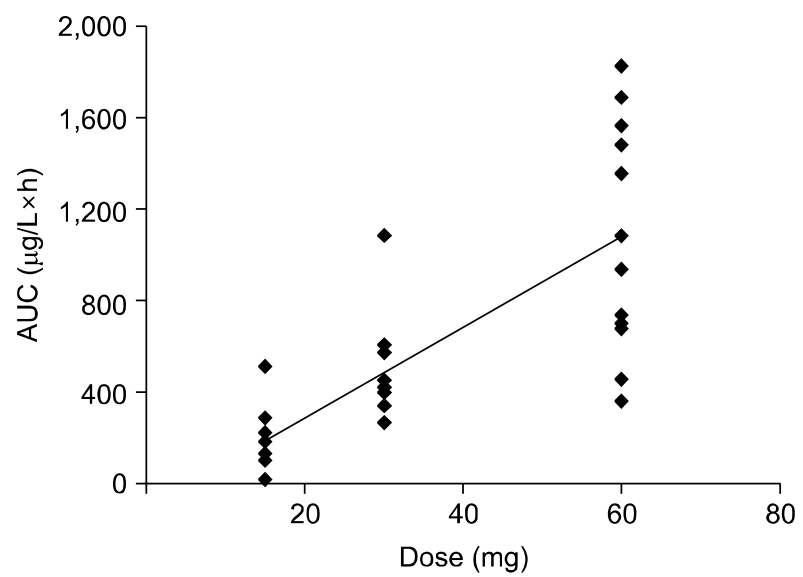

B

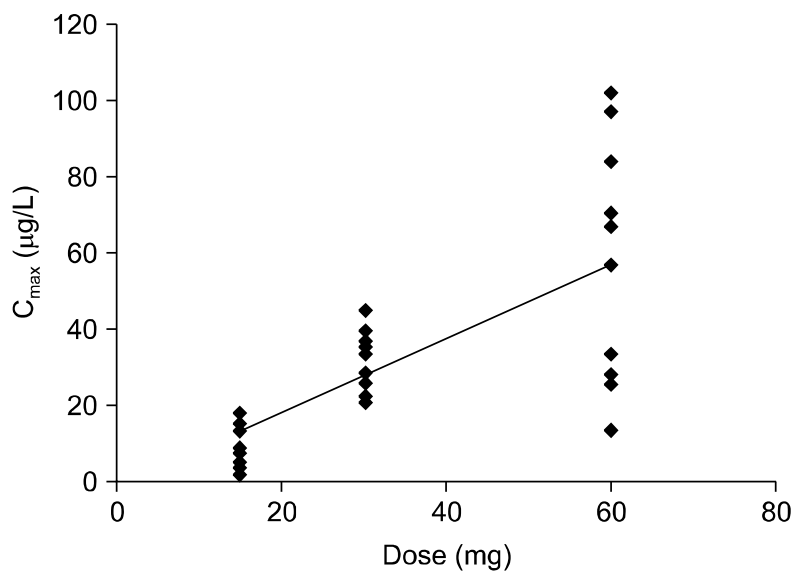

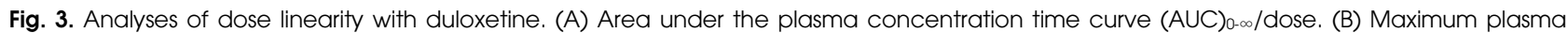
concentration $\left(\mathrm{C}_{\max }\right) /$ dose.

of the slopes were $0.980-1.300$ for $\mathrm{C}_{\max }$ and 19.900-25.638 for $\mathrm{AUC}_{0-\infty}(p<0.000)$. Subjects in the middle dose group in the single-dose phase of the PK study continued to the multiple-dose phase of the study and received $30 \mathrm{mg}$ duloxetine for 7 days. The PK agreed with the single-compartment model. Mean plasma duloxetine concentration-time profiles after multiple oral doses are shown in Fig. 4, and the PK parameters are summarized in Table 1. A steady-state concentration was achieved after administering duloxetine for 3 consecutive days. Mean plasma concentrations on days 4, 5, 6, and 7 before dosing were $13.95 \pm 8.10,16.43 \pm 9.70,17.09 \pm 9.00$, and $17.21 \pm 8.50 \mathrm{ng} / \mathrm{ml}$, respectively. No significant differences in PK parameters were observed between males and females $\left(\mathrm{t}_{1 / 2}: p=0.821 ; \mathrm{Ke}: p=0.144 ; \mathrm{V} 1 / \mathrm{F}: p=0.166\right.$; $\mathrm{CL} / \mathrm{F}: p=0.247 ; \mathrm{AUC}_{0-\mathrm{t}}: p=0.723 ; \mathrm{Ka}: p=0.108 ; \mathrm{T}_{\text {lag }}$ :



Fig. 4. Mean plasma concentration-time curve after multiple dose of $30 \mathrm{mg}$ of duloxetine. 
$p=0.427$ ). Accumulation ratio (the $\mathrm{AUC}_{0-\mathrm{t}}$ on day $7 / \mathrm{AUC}_{0 \text {-t }}$ on day 1$)$ was $1.7 \pm 0.4(1.10 \pm 0.04$ after logarithmic transformation).

\section{Tolerability}

No serious or unexpected adverse events were reported during the PK study. The incidences of adverse events during the single-dose phase were $33.3 \%$ (4/12), $41.7 \%$ $(5 / 12)$, and $66.7 \%(8 / 12)$ in the three dose groups, respectively, and the most common adverse event related to duloxetine was nausea $(12 / 36 ; 33.3 \%)$, followed by dizziness $(6 / 36 ; 16.7 \%)$ and decreased appetite $(3 / 36 ; 8.3 \%)$. A tendency toward an increased incidence of adverse events was observed in the $60 \mathrm{mg}$ dose group, but no difference was found among the three groups $(p=0.250)$. We also observed a case of hypertension in the $60 \mathrm{mg}$ dose group and considered that the drug caused the increase, so we terminated the subject from the study. The most common adverse events in the multiple-dose phase were nausea $(6 / 12 ; 50 \%)$ and dizziness $(2 / 12 ; 16.7 \%)$. All adverse events were mild or moderate in intensity and disappeared after discontinuation of duloxetine. The results of vital signs, ECG, clinical laboratory assessments, and physical examinations were within normal limits for all subjects, and no clinically significant differences were detected among the groups during the study.

\section{DISCUSSION}

Duloxetine was approved for treating adults with MDD in 2004 and is also used to treat pain related to diabetic peripheral neuropathy and stress urinary incontinence. The primary purpose of this study was to assess the safety, tolerability, and PK of duloxetine after administration of single and multiple oral doses in Chinese healthy volunteers.

The 15, 30, and $60 \mathrm{mg}$ oral doses were used in the single-dose phase and agreed with the one-compartment PK model. Serum duloxetine $\mathrm{C}_{\max }$ and AUC increased linearly after administration. A steady state was reached after administering duloxetine for 3 consecutive days in the multiple-dose phase, in agreement with the one-compartment PK model. No differences in $\mathrm{T}_{\max }$ or $\mathrm{t}_{1 / 2}$ values were observed between the single- and multiple-dose phases. The AUC in steady-state $\left(\mathrm{AUC}_{\mathrm{ss}}\right)$ was approximately 1.7 times that of the single-dose phase, indicating an accumulation of duloxetine after multiple-dose administration.

The duloxetine doses used in this study were clinically recommended doses, gender had no significant effect on the PK parameters, and the results agreed with those from another report. ${ }^{9)}$ The main PK results were consistent with a study conducted by Zhao et al. ${ }^{10)}$ in Chinese healthy volunteers. Chan et al. ${ }^{11)}$ compared the single- and multiple-dose PK of duloxetine between healthy Japanese and Caucasians and found no significant difference in $\mathrm{C}_{\max }$, AUC, or $t_{1 / 2}$. Our results were similar to those of the Japanese, suggesting that the PK properties of duloxetine are similar between races.

Duloxetine is extensively metabolized in the liver by CYP1A2 and CYP2D6. ${ }^{5)}$ The variability in AUC and $\mathrm{C}_{\max }$ values may due to different CYP1A2 and CYP2D6 isozyme phenotypes. ${ }^{11)}$ The AUC and $\mathrm{C}_{\max }$ coefficients of variation ranged from $42.24 \%$ to $51.32 \%$ and from $23.76 \%$ to $52.30 \%$, respectively, in this study; however, differences in CYP1A2 and CYP2D6 activity are difficult to detect in a clinical study. The coefficient of variation also indicates the importance of monitoring blood concentrations and preparing individualized doses to ensure safety and effectiveness.

In conclusion, duloxetine administered orally to Chinese healthy volunteers at $15-60 \mathrm{mg}$ was safe and well tolerated. The PK properties of duloxetine were linear. The AUC and $\mathrm{C}_{\max }$ were higher in the multiple-dose phase than in the single-dose phase, indicating that duloxetine accumulated following multiple-dose administration of $30 \mathrm{mg}$. Gender had no significant effect on the PK parameters.

\section{- Acknowledgments}

This study was supported by National Major Project for IND, Clinical tech-platform for evaluation of new drug in psychiatry (No. 2012ZX09303-003) and Shanghai Health talent professional project (No. XBR2011049).

\section{REFERENCES}

1. Westanmo AD, Gayken J, Haight R. Duloxetine: a balanced and selective norepinephrine- and serotonin-reuptake inhibitor. Am J Health Syst Pharm 2005;62:2481-2490.

2. Waitekus AB, Kirkpatrick P. Duloxetine hydrochloride. Nat Rev Drug Discov 2004;3:907-908.

3. Cruz MP, Gonzales ME, Jacobs J, LaFave MC. Duloxetine HCI (Cymbalta) for the treatment of depression, neuropathic pain, fibromyalgia, and stress urinary incontinence. Drug Forecast 2006;31:84-97.

4. Skinner MH, Kuan HY, Pan A, Sathirakul K, Knadler MP, Gonzales CR, et al. Duloxetine is both an inhibitor and a substrate of cytochrome P4502D6 in healthy volunteers. Clin Pharmacol Ther 2003;73:170-177.

5. Lantz RJ, Gillespie TA, Rash TJ, Kuo F, Skinner M, Kuan $\mathrm{HY}$, et al. Metabolism, excretion, and pharmacokinetics of duloxetine in healthy human subjects. Drug Metab Dispos 2003;31:1142-1150.

6. Bymaster FP, Beedle EE, Findlay J, Gallagher PT, Krushin- 
ski JH, Mitchell S, et al. Duloxetine (Cymbalta), a dual inhibitor of serotonin and norepinephrine reuptake. Bioorg Med Chem Lett 2003;13:4477-4480.

7. Eli Lilly and Co. Cymbalta (duloxetine) product information. [cited 2012 Jul]. Available from http://pi.lilly.com/us/ cymbalta-pi.pdf.

8. Sharma A, Goldberg MJ, Cerimele BJ. Pharmacokinetics and safety of duloxetine, a dual-serotonin and norepinephrine reuptake inhibitor. J Clin Pharmacol 2000;40:161167.
9. Guo XF, Zhao JP, Chen JD. Duloxetine: a novel antidepressant. Chin J New Drugs Clin Rem 2006;25:552-555.

10. Zhao RK, Cheng G, Tang J, Song J, Peng WX. Pharmacokinetics of duloxetine hydrochloride enteric-coated tablets in healthy Chinese volunteers: a randomized, open-label, singleand multiple-dose study. Clin Ther 2009;31:1022-1036.

11. Chan C, Yeo KP, Pan AX, Lim M, Knadler MP, Small DS. Duloxetine pharmacokinetics are similar in Japanese and Caucasian subjects. Br J Clin Pharmacol 2007;63:310-314. 